\title{
A Phase Reconstruction Algorithm From Bispectrum
}

\author{
SALEH ALSHEBEILI AND A. ENIS ÇETIN, MEMBER, IEEE
}

\begin{abstract}
A new computationally efficient procedure for the reconstruction of the impulse response of a (minimum or nonminimum phase) linear time-invariant (LTI) system from its bispectrum is presented. This method is based on computing the cepstrum of the impulse response sequence from the $\omega_{1}=\omega_{2}$ slice of the bispectrum. The algorithm can be implemented by using only the one-dimensional fast Fourier transform algorithm.
\end{abstract}

\section{INTRODUCTION}

$\mathrm{M}$ ETHODS BASED on higher order spectra [1], [2], defined as the Fourier transform of the corresponding higher order cumulant, have recently been applied to many problems in a number of diverse fields, including geophysical signal processing [3] and optics [4].

In some seismic signal processing problems it is assumed that the earth consists of horizontal and uniform layers that transmit and reflect the seismic wavelet which is due to a surface energy source. The seismic reflection data $\{x(k)\}$ can be modeled by using Wold's decomposition theorem as

$$
x(k)=\sum_{l=0}^{M} h(l) w(k-l)
$$

where $\boldsymbol{h}=\{h(n)\}_{n=0}^{M}$ is the seismic wavelet, and $\{w(k)\}$ is the reflection coefficient sequence of the layered earth.

In order to deconvolve the reflection coefficients $\{w(k)\}$ from observations $\{x(k)\}$, the wavelet $\boldsymbol{h}$ has to be estimated. Lii and Rosenblatt [5] proposed a method of computing the phase of $\boldsymbol{h}$ from the bispectrum of $\{x(k)\}$. In this approach $\{w(k)\}$ is assumed to have nonzero third-order cumulants, and to be white.

In this paper we present a computationally efficient algorithm to reconstruct the phase of a (minimum or nonminimum phase) linear time-invariant system from its bispectrum. Several techniques have been proposed to solve this problem [1]-[9]. (See the review papers [2] and [3] for the discussion of the other related reconstruction methods.) Recently, Pan and Nikias [6] developed a phase reconstruction method from bispectrum by using homo-

Manuscript received June 13, 1989; revised October 2, 1989. This work was supported by Bilkent University. Ankara, Turkey, and by the National Scientific and Engineering Research Council (NSERC) of Canada. This work was partially presented at the 14 th Biennial Symposium on Communications held at the Queens University, Kingston, ON, Canada.

$\mathrm{S}$. Alshebeili is with the Department of Electrical Engineering, University of Toronto. Toronto, ON M5S 1 A4. Canada.

A. E. Cetin is with the Department of Electrical and Electronics Engineering. Bilkent University. Bilkent, Ankara. Turkey.

IEEE Log Number 8933251. morphic signal-processing techniques. In the new phase reconstruction method we also use cepstral domain methods.

\section{Reconstruction Problem}

In this section we present the phase reconstruction problem. Let $\{x(k)\}$ be a random process which is generated as follows:

$$
x(k)=\sum_{l=-N}^{M} h(l) w(k-l)
$$

where $\{w(k)\}$ is non-Gaussian, white, i.i.d. with $E[w(k)]=0, E[w(k) w(k+n)]=\alpha \delta_{1}(n)$, and $E[w(k) w(k+\tau) w(k+\rho)]=\beta \delta_{2}(\tau, \rho) ; \delta_{1}(n)$ and $\delta_{2}(\tau, \rho)$ are one- and two-dimensional unit impulse sequences, respectively; the sequence $\boldsymbol{h}=\{h(n)\}_{n=-N}^{M}$, where $N$ and $M$ are positive integers, is the system impulse response. The two-dimensional cumulant sequence of the random process $\{x(k)\}$ is defined as follows:

$$
r_{x}\left(n_{1}, n_{2}\right)=E\left[x(k) x\left(k+n_{1}\right) x\left(k+n_{2}\right)\right] .
$$

The bispectrum $B_{x}\left(\omega_{1}, \omega_{2}\right)$ of the random process $\{x(k)\}$ is defined as the Fourier transform of the two-dimensional cumulant sequence; i.e.,

$$
\begin{aligned}
B_{x}\left(\omega_{1}, \omega_{2}\right)= & \sum_{n_{1}=-\infty}^{\infty} \sum_{n_{2}=-\infty}^{\infty} r_{x}\left(n_{1}, n_{2}\right) \\
& \cdot \exp \left(-j\left(\omega_{1} n_{1}+\omega_{2} n_{2}\right)\right) .
\end{aligned}
$$

In this paper we consider the problem of reconstructing the impulse response sequence $\boldsymbol{h}$ from the bispectrum $B_{x}\left(\omega_{1}, \omega_{2}\right)$.

It can be shown that [1], [2]

$$
B_{x}\left(\omega_{1}, \omega_{2}\right)=\beta H\left(\omega_{1}\right) H\left(\omega_{2}\right) H^{*}\left(\omega_{1}+\omega_{2}\right)
$$

where $H(\omega)$ is the Fourier transform of $\boldsymbol{h}$; i.e.,

$$
\mathcal{F}[\boldsymbol{h}](\omega) \triangleq H(\omega)=\sum_{n=-N}^{M} h(n) \exp (-j \omega n)
$$

and $\beta=E\left[w(k)^{3}\right]$. We will use this result (6), which is proved by Brillinger, in our reconstruction algorithm.

\section{Phase Reconstruction Method from BISPECTRUM}

In this section we present a new signal reconstruction method from bispectrum by using homomorphic signal processing [10]. Pan and Nikias [6] developed a complex cepstrum-based signal reconstruction method by obtaining a convolutional relation between the cumulant se- 
quence of the random process $\{x(n)\}$ and the two-dimensional complex cepstrum of the cumulant sequence. Our method is based on the computation of the one-dimensional complex cepstrum corresponding to $B_{x}\left(\omega_{1}\right.$, $\left.\omega_{2}\right)\left.\right|_{\omega_{1}=\omega_{2}=\omega}$.

The new signal reconstruction algorithm from bispectrum is based on the following observations: The complex cepstrum $\hat{\boldsymbol{h}}$ of the sequence $\boldsymbol{h}$ is defined as the inverse $z$-transform of $\log \left(H_{z}(z)\right)$; i.e.,

$$
\hat{h}=Z^{-1}\left[\log H_{z}(z)\right]
$$

jwhere $Z^{-1}$ is the inverse $z$-transform operator and $H_{z}(z)$ is the $z$-transform of $\boldsymbol{h} . H_{z}(z)$ is defined as follows:

$$
H_{z}(z)=\sum_{n=-\infty}^{\infty} h(n) z^{-n} \text {. }
$$

Let us define a sequence, $g=\{g(n)\}$, as follows:

$$
g=F^{-1}[G(\omega)]
$$

where $G(\omega)=\left.B_{x}\left(\omega_{1}, \omega_{2}\right)\right|_{\omega_{1}=\omega_{2}=\omega}$ and $\mathcal{F}^{-1}$ is the inverse Fourier transform operator. The complex cepstrum $\hat{g}(n)$ of the sequence $g(n)$ is given by

$$
\hat{\mathbf{g}}=Z^{-1}\left[\log G_{-}(z)\right]
$$

where $G_{z}(z)$ is the $z$-transform of $g$. If $G_{z}(z)$ has no zeros on the unit circle, then

$$
\hat{g}=\mathcal{F}^{-1}[\log G(\omega)]=\mathfrak{F}^{-1}\left[\log B_{x}(\omega, \omega)\right] .
$$

By using (5) we obtain the following equation:

$$
\begin{aligned}
\log B_{x}(\omega, \omega)= & \log H(\omega)+\log H(\omega) \\
& +\log H^{*}(2 \omega)+\log \beta .
\end{aligned}
$$

The inverse Fourier transform of $\log H(\omega)$ is $\hat{\boldsymbol{h}}$, and the inverse Fourier transform of $\log H^{*}(2 \omega)$ is the following sequence:

$$
\mathcal{F}^{-1}\left[\log H^{*}(2 \omega)\right]= \begin{cases}\hat{h}(-n / 2), & \text { if } n \text { is even } \\ 0, & \text { if } n \text { is odd }\end{cases}
$$

By using (12) and (13) one can show that the complex cepstrum $\hat{g}$ of the sequence $g$ is given by

$$
\hat{g}(n)= \begin{cases}3 \hat{h}(0)+\log \beta, & \text { if } n=0 \\ 2 \hat{h}(n)+\hat{h}(-n / 2), & \text { if } n \text { is even, } n \neq 0 \\ 2 \hat{h}(n), & \text { if } n \text { is odd. }\end{cases}
$$

The complex cepstrum of the impulse response sequence $\hat{h}(n)$ can be recursively obtained from $\hat{g}(n)$; i.e.,

$$
\begin{aligned}
\hat{h}(0) & =(\hat{g}(0)-\log \beta) / 3, \quad \hat{h}(1)=\hat{g}(1) / 2, \\
\hat{h}(-1) & =\hat{g}(-1) / 2, \hat{h}(2)=(\hat{g}(2)-\hat{h}(-1)) / 2, \\
\hat{h}(-2) & =(\hat{g}(-2)-\hat{h}(1)) / 2, \hat{h}(3)=\hat{g}(3) / 2, \\
\hat{h}(-3) & =\hat{g}(-3) / 2, \ldots
\end{aligned}
$$

or

$\hat{h}(n)= \begin{cases}(\hat{g}(0)-\log \beta) / 3, & \text { if } n=0 \\ (\hat{g}(n)-\hat{h}(-n / 2)) / 2, & \text { if } n \text { is even, } n \neq 0 \\ \hat{g}(n) / 2, & \text { if } n \text { is odd. }\end{cases}$

After obtaining the sequence $\hat{h}(n)$, the sequence $h(n)$ can be obtained by using the definition of the complex cepstrum; i.e.,

$$
\boldsymbol{h}=\mathfrak{F}^{-1}[\exp (\mathfrak{F}[\hat{h}])] .
$$

In view of the above observations, the reconstruction algorithm consists of the following steps:

1) Estimate the two-dimensional cumulant sequence $r_{x}\left(n_{1}, n_{2}\right)$.

2) Compute $g(n)$, which is defined in (9) as follows:

$$
g(n)=\sum_{l} r_{x}(l, n-l)
$$

Equation (18) can easily be proved [11].

3) Compute $G(\omega)=\mathscr{F}[g(n)]$ and $\hat{G}(\omega)=\log$ $G(\omega)$.

4) Compute $\{\hat{g}(n)\}=\mathscr{F}^{-1}[\hat{G}(\omega)]$ and obtain $\hat{h}(n)$ from $\hat{g}(n)$ by using (15)

5) Obtain $h(n)$ from $\hat{h}(n)$ by using (17).

The Fourier transform operations in steps 3-5 can be carried out by using a fast Fourier transform (FFT) algorithm.

In practice, $\beta$ is not known, thus the reconstruction of the impulse response sequence is possible only to a constant factor. Also, the complex cepstra of $h(n)$ and $h(n$ $-K)$, where $K$ is any integer, are equal to each other. Thus the reconstructed sequence may be a shifted and scaled version of the original sequence.

In general, the complex cepstrum $\hat{y}(n)$ of a finite extent sequence $y(n)$ is of infinite extent. Thus both $\hat{g}(n)$ and $\hat{h}(n)$ are probably infinite sequences. However, the cepstral sequences decay exponentially with a $1 / n$ scaling factor for finite extent sequences [10]. Thus we can select two large integers, $p>0$ and $q<0$, such that the truncated sequence $\hat{\boldsymbol{h}}_{T}=\{\cdots, 0,0, \hat{h}(q), \hat{h}(q+1)$, $\cdots \hat{h}(0), \hat{h}(1), \cdots \hat{h}(p), 0,0, \cdots\}$ approximates the sequence $\hat{\boldsymbol{h}}$ arbitrarily closely. In step 4 of the above algorithm, we actually compute a truncated sequence $\hat{\boldsymbol{h}}_{T}$ from $\hat{g}$.

Pan and Nikias's method [6] is dependent on the selection of integers $p$ and $q$. In our method, the integers $p$ and $q$ can be easily selected because the sequence $\hat{g}(n)$ is available. Also, in our algorithm we use a one-dimensional complex cepstrum.

In order to obtain the cepstrum $\hat{g}(n)$ from $G(\omega)$, onedimensional phase unwrapping is necessary. We used Quatieri and Tribolet's algorithm [12] to unwrap the phase of $G(\omega)$. A similar signal-reconstruction algorithm, based 
on the differential cepstrum, which does not require phase unwrapping is also developed in the next section. Also, we assumed that $H(\omega) \neq 0$ for all $\omega \in[-\pi, \pi]$, otherwise the cepstra $\hat{h}(n), \hat{g}(n)$ should be computed on a circle on which $H_{z}(z)$ has no zeros in the $z$ domain.

We can also obtain the minimum and maximum phase components of the impulse response sequence $\boldsymbol{h}$. Let $\boldsymbol{h}_{t}$ and $\boldsymbol{h}_{r}$ be the minimum and maximum phase components of the sequence $\boldsymbol{h}$, respectively. The sequences $\boldsymbol{h}_{l}$ and $\boldsymbol{h}_{r}$ satisfy the following relation:

$$
\boldsymbol{h}=\boldsymbol{h}_{l} * \boldsymbol{h}_{r}
$$

and the $z$ transform of $\boldsymbol{h}_{l}\left(\boldsymbol{h}_{r}\right)$ has no zeros (outside) inside the unit circle. The complex cepstrum of $\boldsymbol{h}$ satisfies the following equation [6]:

$$
\hat{h}=\hat{\boldsymbol{h}}_{l}+\hat{\boldsymbol{h}}_{r}
$$

where $\hat{\boldsymbol{h}}_{l}$ and $\hat{\boldsymbol{h}}_{r}$ are the cepstra of $\boldsymbol{h}_{l}$ and $\boldsymbol{h}_{r}$, respectively. Furthermore, $\hat{\boldsymbol{h}}_{l}\left(\hat{\boldsymbol{h}}_{r}\right)$ is causal (anti-causal). Thus,

$$
\hat{\boldsymbol{h}}_{l}=\{\cdots 0,0, \hat{h}(0) / 2, \hat{h}(1), \hat{h}(2), \cdots\}
$$

and

$$
\hat{\boldsymbol{h}}_{r}=\{\cdots \hat{h}(-2), \hat{h}(-1), \hat{h}(0) / 2,0,0, \cdots\} .
$$

Recently, Dianat and Raghuveer [13] also developed a reconstruction algorithm by using the slice $B_{x}(\omega, \omega)$ of the bispectrum. The algorithm in [13] is different from ours.

\section{Reconstruction Method Based on Differential Cepstrum}

In this section we develop a phase reconstruction algorithm by using differential cepstrum [14]. This algorithm is very similar to the method described in Section III.

The differential cepstrum $v_{d}(n)$ of a sequence $v(n)$ is defined as follows:

$$
v_{d}(n)=Z^{-1}\left[\frac{1}{V_{z}(z)} \frac{\partial V_{z}(z)}{\partial z}\right]
$$

The cepstrum $\hat{v}(n)$ of $v(n)$ is related to the differential cepstrum as follows:

$$
\hat{v}(n)= \begin{cases}-v_{d}(n+1) / n, & \text { if } n \neq 0 \\ 0, & \text { if } n=0\end{cases}
$$

By using (14) and (24) we obtain the relation between $g_{d}(n)$, the differential cepstrum of $g(n)$, and $h_{d}(n)$, the differential cepstrum of $h(n)$; i.e.,

$$
g_{d}(n)= \begin{cases}2 h_{d}(n)-2 h_{d}((3-n) / 2), & \text { if } n \text { is odd } \\ 2 h_{d}(n), & \text { if } n \text { is even. }\end{cases}
$$

The differential cepstrum $h_{d}(n)$ of $h(n)$ can be recursively obtained from $g_{d}(n)$ as in the previous section; i.e.,

$$
\begin{array}{r}
h_{d}(0)=g_{d}(0) / 2, \quad h_{d}(1)=0, \quad h_{d}(2)=g_{d}(2) / 2, \\
h_{d}(-1)=\left(g_{d}(-1)+2 h_{d}(2)\right) / 2, \\
h_{d}(3)=\left(g_{d}(3)+2 h_{d}(0)\right) / 2, \quad h_{d}(4)=g_{d}(4) / 2, \\
h_{d}(-3)=\left(g_{d}(-3)+2 h_{d}(3)\right) / 2, \cdots
\end{array}
$$

or

$$
h_{d}(n)=\left\{\begin{aligned}
{\left[g_{d}(n)+\right.} & \left.2 h_{d}((3-n) / 2)\right] / 2 \\
& \text { if } n \text { is odd } \\
g_{d}(n) \quad & \text { if } n \text { is even. }
\end{aligned}\right.
$$

The sequence $\hat{h}(n)$ is obtained from $h_{d}(n)$ by using (24), and $h(n)$ is calculated from $\hat{h}(n)$ as described in the previous section.

Computation of the differential cepstrum does not require the phase unwrapping which is necessary to compute the cepstrum of a sequence.

The differential cepstrum of $c h(n-K)$, where $c$ is any nonzero real number and $K$ is an arbitrary integer, is the same as the differential cepstrum of $h(n)$. Because of this fact the reconstructed sequence may be a shifted and scaled version of the original sequence.

Pan and Nikias's FFT-based method [6] requires 3 twodimensional FFT computations to obtain the two-dimensional cepstrum $\hat{r}_{x}\left(n_{1}, n_{2}\right)$ from the $r_{x}\left(n_{1}, n_{2}\right)$ sequence. After this step, the minimum and maximum phase components $\hat{\boldsymbol{h}}_{/}$and $\hat{\boldsymbol{h}}_{r}$ are obtained recursively from $\hat{\boldsymbol{r}}_{x}\left(n_{1}, n_{2}\right)$. In our algorithm the differential cepstrum $g_{d}(n)$ can be obtained from $r_{x}\left(n_{1}, n_{2}\right)$ by three one-dimensional operations, and $\hat{h}(n)$ (or equivalently minimum and maximum phase components) is recursively obtained from $g_{d}(n)$ by using (26) and (27). Thus our algorithm is more computationally efficient than Pan and Nikias's FFT-based method.

\section{Simulation Examples}

In this section we present some simulation examples, in which the LTI systems are driven by noise which is zero-mean, exponentially distributed, white, and was generated by the GGEXN subroutine of the IMSL library, with $\beta=2$.

\section{Example 1}

In this example, the LTI minimum phase system $H(z)$ $=\left(1-0.35 z^{-1}\right)\left(1-0.5 z^{-1}\right)=1-0.85 z^{-1}+$ $0.175 z^{-2}$ is used. The third-order cumulant sequence is estimated as in [6]. The sequence $g(n)$ is computed by using (18). An FFT of size 128 is used to compute $G(\omega)$ at 128 frequency points. The $p$ and $q$ values are chosen to be 32. Three different lengths of output data have been used for this example: $1 \times 128,8 \times 128$, and $16 \times 128$. 
The impulse response sequence is determined as the sample mean of 100 different output data realizations. The reconstructed impulse response sequences are shown in Table I. Comparisons with Pan and Nikias's method [6] are also presented in Table I.

\section{Example 2}

In this example, the nonminimum phase system $H(z)$ $=\left(1-0.35 z^{-1}\right)(1-0.5 z)=-0.5 z+1.175-$ $0.35 z^{-1}$ is used. This system is a mixed-phase moving average (MA) system. We estimated $G(\omega)$ as in the previous example. The $p$ and $q$ values are also chosen to be 32. We again used three different lengths of output data in this example: $1 \times 128,8 \times 128$, and $16 \times 128$. The impulse response sequence is determined as the sample mean of 100 different output data realizations. The reconstructed impulse response sequences are shown in Table II. Comparisons with Pan and Nikias's method [6] are also presented in Table II.

\section{Example 3}

In this example, the MA system $H(z)=(1-0.85$ $\left.z^{-1}\right)\left(1-1.2 z^{-1}+0.45 z^{-2}\right)(1-0.869 z)(1-1.1$ $\left.z+0.617 z^{2}\right)$ is used. The impulse response sequence of this system is shown in Fig. 1. This system has zeros which are close to the unit circle. We used output data sequences of length $10 \times 128$. The impulse response sequence is determined as the sample mean of 128 different output data realizations. The reconstructed impulse response sequence is also shown in Fig. 1. If the system has zeros on the unit circle, then the cepstral sequences $\hat{h}(n)$ and $\hat{g}(n)$ can still be computed by obtaining the inverse $z$-transform of $G_{z}(z)$ on a circle on which $H_{z}(z)$ has no zeros. The impulse response of the ARMA systems can also be approximately obtained by using this algorithm.

\section{Example 4}

In this example, the LTI system is the following ARMA system: $H(z)=\left(1-0.35 z^{-1}\right)(1-0.5 z) /(1-0.2$ $\left.z^{-1}\right)$. The impulse response sequence of this system is $h$ $=\{\cdots 0,0, h(-1)=-0.5,1.075,-0.135,-0.03$, $\cdots\}$. An FFT of size 128 is used to compute $G(\omega)$ at 128 frequency points. The $p$ and $q$ values are chosen to be 32 . We used output data sequences of length $10 \times 128$. The impulse response sequence is determined as the sample mean of 128 different output data realizations. The reconstructed impulse response sequence is $\{\cdots$ $-0.003,-0.504,1.076,-0.1348,-0.025, \cdots\}$.

In general, we observed that our method produces comparable results to Pan and Nikias's method [6]. This is because of the fact that both methods are based on the computation of the cepstrum of the system impulse response.

\section{CONCLUSION}

In this paper, a new procedure for reconstructing the impulse response of a minimum or nonminimum phase linear time-invariant system from its bispectrum is pro-

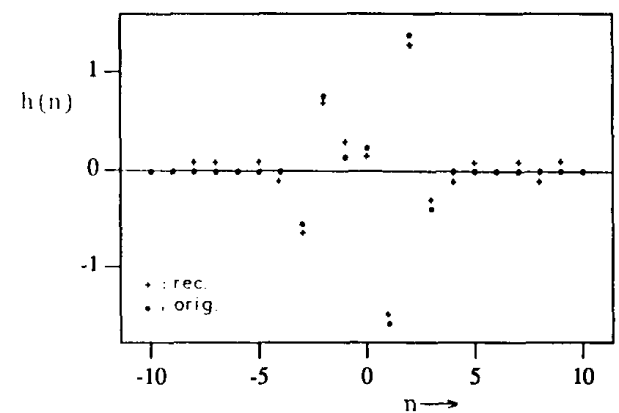

Fig. 1. The true and the reconstructed impulse response sequences in Example 3.

TABLE I

Thf Reconstruction of the IMPUlse Response Sequence of The System WHICH IS DESCRIBED IN EXAMPLE 1

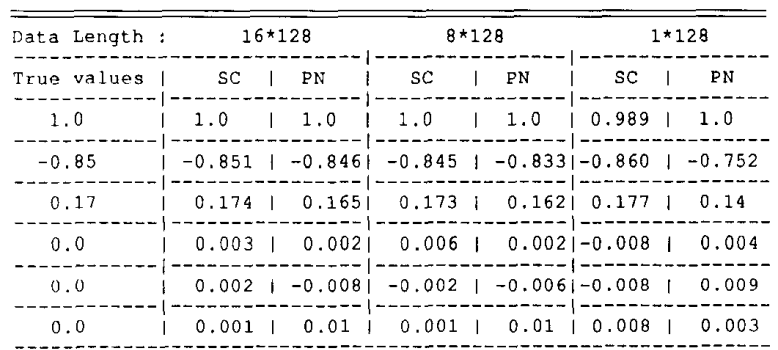

PN stands for Pan and Nikias's method and SC is the method described in this paper.

TABLE II

THE ReCONSTRUCtion of THE IMPULSE RESPONSE SEQUENCE OF THE SYSTEM WhICH IS DESCRIBED IN EXAMPLE 2

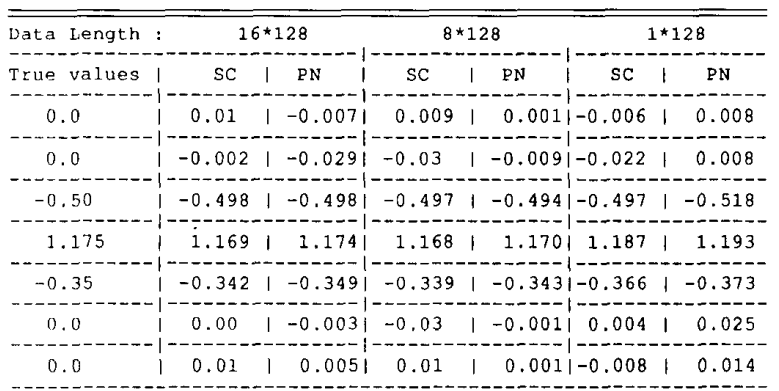

PN stands for Pan and Nikias's method and SC is the method described in this paper.

posed. This method is based on computing the cepstrum of the impulse response sequence from the $\omega_{1}=\omega_{2}$ slice of the bispectrum. The new method is a computationally efficient procedure and can be implemented by using only one-dimensional fast Fourier transform computations.

\section{REFERENCES}

[1] D. R. Brillinger, "An introduction to polyspectra," Ann. Math. Stat. vol. 36, pp. $1351-1374,1965$

[2] C. L. Nikias and M. R. Raghuveer, "Bispectrum estimation: A digital signal processing framework," Proc. IEEE, vol. 75, pp. 869$891,1987$. 
[3] T. Matsuoka and T. J. Ulrych, "Phase estimation using the bispectrum," Proc. IEEE, vol. 72, pp. 1403-1411, 1984.

[4] A. W. Lohmann and B. Wirnitzer. "The triple correlations," Proc. IEEE, vol. 72, pp. 889-901, 1984

[5] K. S. Lii and M. Rosenblatt, "Deconvolution and estimation of transfer function phase and coefficients for non-Gaussian linear processes," Ann. Stat., vol. 10, pp. 1195-1208, 1982.

[6] R. Pan and C. L. Nikias, "The complex cepstrum of higher order cumulants and nonminimum-phase system identification," IEEE Trans. Acoust., Speech, Signal Processing, vol. 36, pp. 186-206 1988.

[7] J. K. Tugnait, "Identification of nonminimum-phase linear stochastic systems," Automatica, vol. 22, pp. 457-464, 1986.

[8] G. B. Giannakis, "Cumulants: A powerful tool in signal processing,"' Proc. IEEE, vol. 75, pp. 1333-1334, 1987.

[9] G. B. Giannakis and J. Mendel, "Identification of nonminimum-phase systems using higher order statistics," IEEE Trans. Acoust., Speech, Signal Processing, vol. 36, pp. 186-206, 1989.

[10] A. V. Oppenheim and R. W. Schafer, Digital Signal Processing. Englewood Cliffs, NJ: Prentice-Hall, 1975, ch. 10.

[11] D. Dudgeon and R. Merserau, Multidimensional Digital Signal Processing. Englewood Cliffs, NJ: Prentice-Hall, 1984

[12] T. F. Quatieri and J. M. Tribolet, "Computation of the real cepstrum and the minimum-phase reconstruction," Programs for Digital Signal Processing. New York: IEEE, 1979, ch. 7

[13] S. A. Dianat and M. R. Raghuveer, "Bispectrum phase transformation for nonminimum phase signal reconstruction," in Proc. ICASSP'89 (Glasgow, Scotland), pp. 1275-1277.

[14] A. Polydoros and A. Fam, "The differential cepstrum: Definition and properties," in Proc. IEEE Int. Symp. Circuits and Syst., Apr. 1981, pp. $77-80$.
Saleh Alshebeili, photograph and biography not available at the time of publication.

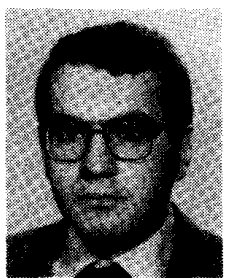

A. Enis Çetin ( $\left.\mathrm{S}^{\prime} 84-\mathrm{M}^{\prime} 87\right)$ received the B.Sc. degree in electrical engineering from the Middle East Technical University. Ankara, Turkey, in 1984, and the M.S.E. and Ph.D. degrees from the Moore School of Electrical Engineering. University of Pennsylvania, Philadelphia, in 1986 and 1987 , respectively.

In 1987 he became Assistant Professor of Electrical Engineering at the University of Toronto. During the summer of 1988 he was a Consultant at the Bell Communications Research. Morristown, NJ. He is now with the Bilkent University. Ankara. His research interests are primarily in digital and statistical signal processing. 